\title{
A conserved role for the ARC1 E3 ligase in Brassicaceae self-incompatibility
}

\author{
Emily Indriolo ${ }^{1}$ and Daphne R. Goring ${ }^{1,2}$ * \\ ' Department of Cell \& Systems Biology, University of Toronto, Toronto, ON, Canada \\ ${ }^{2}$ Centre for the Analysis of Genome Evolution \& Function, University of Toronto, Toronto, ON, Canada
}

\author{
Edited by: \\ Hongyong Fu, Institute of Plant and \\ Microbial Biology, Academia Sinica, \\ Taiwan \\ Reviewed by: \\ Yongbiao Xue, Institute of Genetics \\ and Developmental Biology, Chinese \\ Academy of Sciences, China \\ James Doughty, University of Bath, \\ UK \\ ${ }^{*}$ Correspondence: \\ Daphne R. Goring, Department of Cell \\ \& Systems Biology, University of \\ Toronto, 25 Willcocks Street, Toronto, \\ ON M5S 3B2, Canada \\ e-mail:d.goring@utoronto.ca
}

Ubiquitination plays essential roles in the regulation of many processes in plants including pollen rejection in self-incompatible species. In the Brassicaceae (mustard family), selfincompatibility drives the rejection of self-pollen by preventing pollen hydration following pollen contact with the stigmatic surface. Self-pollen is recognized by a ligand-receptor pair: the pollen S-locus cysteine rich/S-locus protein 11 (SCR/SP11) ligand and the pistil S receptor kinase (SRK). Following self-pollen contact, the SCR/SP11 ligand on the pollen surface binds to SRK on the pistil surface, and the SRK-activated signaling pathway is initiated. This pathway includes the armadillo repeat containing 1 (ARC1) protein, a member of the plant U-box (PUB) family of E3 ubiquitin ligases. ARC1 is a functional E3 ligase and is required downstream of SRK for the self-incompatibility response. This mini review highlights our recent progress in establishing ARC1's conserved role in self-pollen rejection in Brassica and Arabidopsis species and discusses future research directions in this field.

Keywords: ubiquitination, cell signaling, self-incompatibility, Arabidopsis, Brassicaceae

\section{INTRODUCTION}

Plants have evolved complex signaling networks to survive their sessile existence, and protein ubiquitination underpins many of these systems. One process regulated by ubiquitination is selfincompatibility, which prevents the acceptance of self-pollen by the pistil resulting in increased genetic diversity in a population. E3 ubiquitin ligases have been implicated in two different self-incompatibility systems, the S-Ribonuclease-based selfincompatibility (Solanaceae, Rosaceae, and Plantaginaceae) and the $S$ receptor kinase (SRK)-based self-incompatibility (Brassicaceae; reviewed in Hiscock and Allen, 2008; Iwano and Takayama, 2012). This mini review will focus on the role of ubiquitination in the Brassicaceae system that has been well characterized in the Brassica and Arabidopsis species. Species in the Brassicaceae have dry stigmas, and the pollen grain must receive water for hydration from the stigmatic papilla in order to germinate and grow a pollen tube (Heslop-Harrison and Shivanna, 1977). Therefore, when a pollen grain lands on the stigmatic papilla at the top of the pistil in the flower, the stigmatic papilla can determine if the pollen grain should be accepted or rejected. If a pollen grain is determined to be self-incompatible, the stigmatic papilla will reject it by blocking pollen grain hydration and pollen tube growth. Thus, pollen contact at the stigmatic surface is a major regulatory point for pollination (reviewed in Chapman and Goring, 2010).

\section{THE RECEPTOR-LIGAND PAIR REGULATING SELF-INCOMPATIBILITY IN THE BRASSICACEAE}

Initial research in this field was conducted on Brassica species (B. oleracea, B. rapa, B. napus) with the identification of two polymorphic loci regulating self-incompatibility. The Brassica pollen locus encodes the Cysteine Rich/S-locus Protein 11 (SCR/SP11) protein while the Brassica pistil locus encodes the
SRK (Schopfer et al., 1999; Cui et al., 2000; Takasaki et al., 2000; Takayama et al., 2000; Silva et al., 2001). Each specific SCR/SP11$S R K$ allele pair comprises a S-haplotype, whereby recognition causes the rejection of self-pollen to prevent inbreeding, and a number of different Brassica S-haplotypes has been identified (reviewed in Iwano and Takayama, 2012). Sequences for different S-haplotypes (SCR/SP11 and SRK alleles) have subsequently been identified in other Brassicaceae species including Arabidopsis lyrata, Arabidopsis halleri, Arabis alpina, Capsella grandiflora, and a related S-locus region in Leavenworthia alabamica (Kusaba etal., 2001; Schierup etal., 2001; Paetsch etal., 2006; Castric etal., 2008; Boggs etal., 2009; Foxe etal., 2009; Guo et al., 2009; Tedder et al., 2011; Chantha et al., 2013). In Brassica, when a self-pollen grain contacts a stigmatic papilla, the SCR/SP11 ligand from the pollen coat binds to SRK, and SRK becomes autophosphorylated (Giranton et al., 2000; Kachroo et al., 2001; Takayama et al., 2001; Shimosato etal., 2007). As expected, SRK was found to bind strongest to the corresponding SCR/SP11 ligand, but could also bind weakly to other S-haplotype-encoded SCR/SP11 ligands (Kemp and Doughty, 2007; Naithani etal., 2007; Shimosato etal., 2007). There is one known negative regulator of Brassica SRK, Thioredoxin Hlike 1 (THL1; Bower et al., 1996; Cabrillac et al., 2001; Haffani et al., 2004). THL1's inhibition is proposed to prevent SRK from auto-activating and signaling before the recognition of SCR/SP11 at the plasma membrane (Giranton et al., 2000; Cabrillac et al., 2001; Ivanov and Gaude, 2009). After binding of SCR/SP11 to SRK, the self-incompatibility signaling cascade is initiated. This rejection is localized to the point of pollen contact, as a single papilla can simultaneously accept a compatible pollen grain and reject a self-incompatible pollen grain (Dickinson, 1995). 


\section{REGULATORY PROTEINS ACTING DOWNSTREAM OF SRK}

In addition to the role of SCR/SP11 and SRK in mediating initial self-pollen recognition, there are two other proteins that have been identified as positive regulators of the self-incompatibility response in Brassica: the M-locus protein kinase (MLPK; Murase etal., 2004; Kakita etal., 2007b) and the E3 ubiquitin ligase, Armadillo (ARM)-repeat containing 1 (ARC1; Gu et al., 1998; Stone etal., 1999, 2003). As well, more recent research from our group has tied the role of Exo70A1, a key component for polarized exocytosis to be negatively regulated by ARC1 in the self-incompatibility response (Samuel et al., 2009; Safavian and Goring, 2013; Indriolo et al., 2014; Safavian et al., Submitted). B. rapa MLPK is a Receptor-Like Cytoplasmic Kinase (RLCK) that, through alternate splicing, is localized to the plasma membrane via an N-terminal myristoylation site or an N-terminal hydrophobic region, and both forms can complement $m l p k$ mutant stigmatic papillae (Murase et al., 2004; Kakita et al., 2007a). MLPK is proposed to interact with SRK at the plasma membrane, and the SRK-MLPK complex is proposed to phosphorylate downstream signaling proteins (Kakita etal., 2007a,b; Samuel et al., 2008). A. thaliana RLCKs that are closely related to MLPK have been identified, but a corresponding role to MLPK in Arabidopsis self-incompatibility has not been elucidated yet (Kakita et al., 2007a). So far, the only other known downstream component, $\mathrm{ARC1}$, is a member of the Plant U-box (PUB)/ARM repeat family of E3 ligases (Mudgil et al., 2004; Samuel et al., 2006; Yee and Goring, 2009). While ARC1's role in B. napus and A. lyrata self-incompatibility has not been disputed (Stone et al., 1999; Indriolo et al., 2012), some debate does exist as to whether ARC1 is required for reconstituting self-incompatibility in A. thaliana as discussed below (Indriolo et al., 2014). Part of this will likely turn out to be due to the nature of signaling systems using complex multi-branched pathways; as such, one would expect more signaling proteins to be implicated in the SRK pathway in the future.

Plant U-box-armadillo repeat E3 ligases are involved in a wide variety of plant processes including plant-microbe interactions, abiotic stress responses, hormone responses, and development (Mbengue etal., 2010; Lu etal., 2011; Salt et al., 2011; Liu et al., 2012; Seo etal., 2012; Stegmann et al., 2012; Vogelmann et al., 2012; Wang etal., 2013). Several UND-PUB-ARM E3 ligases have been found to interact with receptor kinases (Samuel et al., 2008; Mbengue et al., 2010; Lu et al., 2011). The conserved U-box domain of $\sim 70$ residues was originally identified in yeast UFD2 protein and interacts with the E2 conjugating enzyme (Koegl et al., 1999; Hatakeyama and Nakayama, 2003; Andersen et al., 2004; Schulman and Chen, 2005; Wiborg et al., 2008). A subset of the PUB-ARM proteins, including ARC1, contain a conserved Ubox N-terminal domain (UND; Mudgil et al., 2004; Samuel et al., 2006). The UND domain is proposed to give specificity for proteins ubiquitinated by ARC1 such as the proposed target, Exo70A1, in the instance of self-incompatibility.

\section{ARC1 IS AN E3 LIGASE INVOLVED IN SELF-INCOMPATIBILITY SIGNALING}

Armadillo repeat-containing 1 was originally identified in B. napus, through a yeast two-hybrid screen for SRK kinase domain interactors, and ARC1 was found to bind to SRK through its ARM repeat domain (Gu et al., 1998). ARC1 can be phosphorylated by SRK, but is more strongly phosphorylated by MLPK in vitro (Gu et al., 1998; Samuel et al., 2008). ARC1 is composed of the three distinct protein domains described above (UND, U-box, ARM repeat domain), and has functional nuclear localization and nuclear export signals. When transiently expressed in tobacco BY2 cells, ARC1 was localized to both the cytoplasm and nucleus, shuttling back and forth between these two compartments (Stone et al., 2003). The function of ARC1's nuclear localization is still unclear, especially as it is expected to be near the plasma membrane for its role in self-incompatibility (Figure 1, described in more detail below). When ARC1 was co-expressed with active SRK or MLPK kinase domains, it no longer shuttled to the nucleus suggesting that ARC1 phosphorylation alters its localization and may be important for its function in the self-incompatibility pathway (Stone et al., 2003; Samuel et al., 2008). B. napus ARC1 displays stigma-specific expression, and the knock-down of ARC1 expression by antisense suppression resulted in a gained self-pollen acceptance, instead of rejection, indicating a breakdown in the selfincompatible pathway (Gu et al., 1998; Stone et al., 1999). ARC1 was shown to have in vitro E3 ligase activity, and the importance of ubiquitination in self-incompatibility came from analyses of ubiquitinated proteins in the stigma. Wild-type B. napus stigmas that were pollinated with self-incompatible pollen were shown, by immunoblotting with an anti-ubiquitin antibody, to be enriched in ubiquitinated proteins (Stone et al., 2003; Samuel et al., 2011). In contrast, self-pollinated ARC1-antisense-suppressed stigmas had a lower level of ubiquitinated proteins. Therefore, these data suggest that the presence of ARC1 led to ubiquitinated stigma proteins following self-incompatible pollinations. Given that all this work was done in B. napus, an outstanding question in the field was whether ARC1's function was conserved in other Brassicaceae species.

\section{ARC1 PLAYS A CONSERVED ROLE IN SELF-INCOMPATIBILITY SIGNALING ACROSS THE BRASSICACEAE}

To better understand the Brassicaceae self-incompatibility pathway, one direction of research has examined this trait in Arabidopsis species. A. thaliana lost the self-incompatibility trait due to mutations in the SCR/SRK genes while another Arabidopsis species, A. lyrata, has remained naturally self-incompatible (Kusaba et al., 2001; Schierup etal., 2001). Some A. thaliana ecotypes, such as Wei-1 and Old-1, were actually found to carry intact SRK genes and exhibited self-incompatibility when pollinated with A. halleri pollen expressing the matching SCR ligand (Tsuchimatsu etal., 2010). Self-compatible A. thaliana has been used as an artificial system for reconstructing self-incompatibility by transforming A. lyrata SCR and SRK genes into different A. thaliana ecotypes (Nasrallah et al., 2004; Boggs et al., 2009; Rea et al., 2010; Tsuchimatsu et al., 2010). Interestingly, transgenic A. thaliana expressing SCR and SRK did not always result in the generation of self-incompatibility. Some transgenic ecotypes such as Sha, Kas-2, and C24 were reported to produce self-incompatible flowers, while other transgenic ecotypes such as Col-0, Mt-0, Nd-0, and No remained self-compatible (Nasrallah etal., 2004; Boggs et al., 2009). However, there is ambiguity 


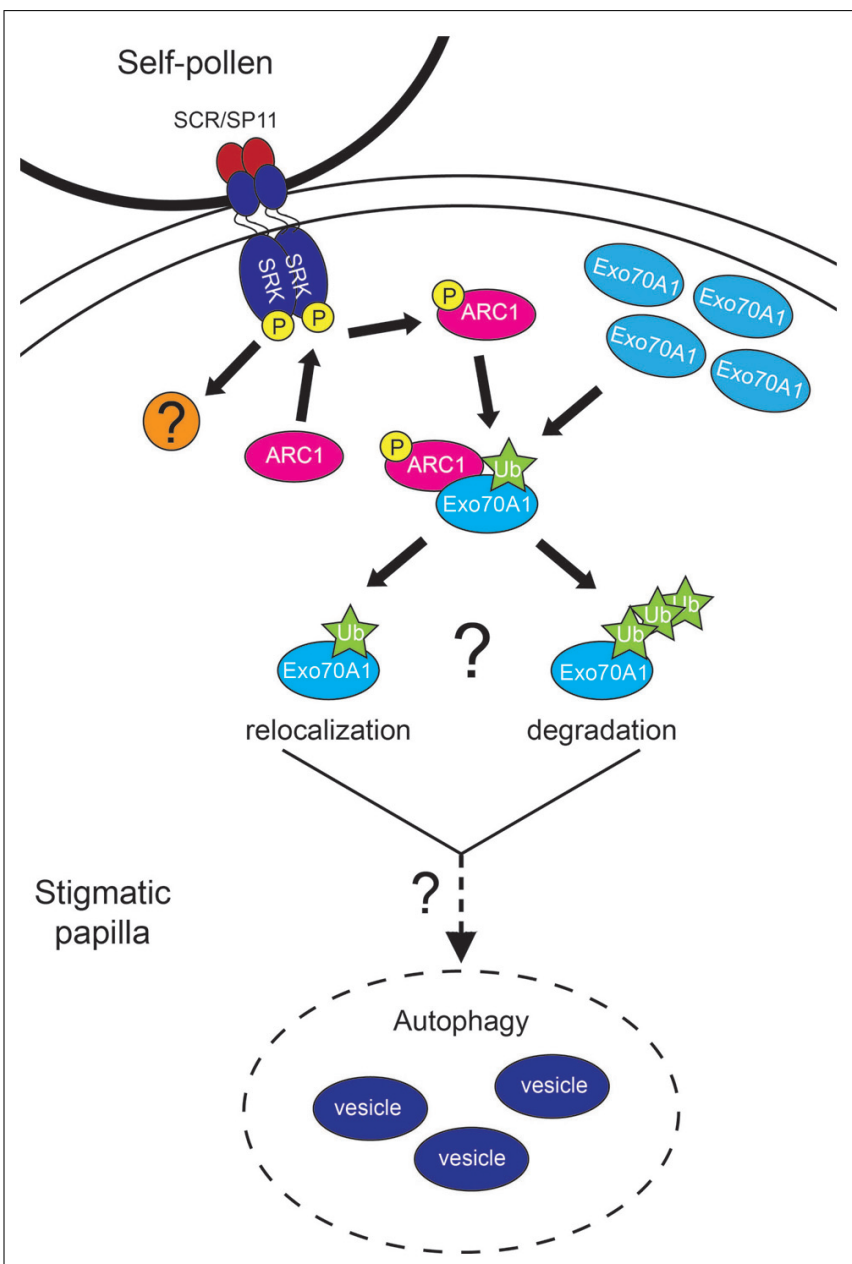

FIGURE 1 | Model for the Self-Incompatibility response in Arabidopsis. Upon pollen contact with the stigmatic papilla, the papilla must determine if the pollen grain is self or non-self. For a self-pollen grain, the SCR/SP11 ligand binds to SRK, and SRK will then become phosphorylated. ARC1 interacts with the phosphorylated cytoplasmic kinase domain of SRK and become phosphorylated and activated. ARC1 then seeks its target for ubiquitination, Exo70A1, which is located at the plasma membrane in preparation for exocyst-mediated exocytosis. ARC1, with the help of an E1 activating enzyme and E2 conjugating enzyme, ubiquitinates Exo70A1. The nature of this ubiquitination is not known, as it could be either mono-ubiqitination resulting in Exo70A1 relocalization, or poly ubiquitination leading to degradation of Exo70A1 by the $26 \mathrm{~S}$ proteosome. Either way, the inhibition of Exo70A1 appears to activate an autophagic response that leads to degradation of the secretory vesicles. There is also another (unknown) factor functioning downstream of SRK in this pathway. How ARC1, this factor, and the ubiquitination of Exo70A1 signals for autophagy is not known. Once the secretory vesicles have been removed, the self-pollen grain will not receive the resources needed for hydration and germination; as a result will be rejected.

in these delineations; for example, transgenic A. thaliana Col0 were sometimes reported as becoming self-incompatible with SCR and SRK expression (Nasrallah et al., 2002; Kitashiba et al., 2011) and other times reported as remaining self-compatible (Nasrallah et al., 2004; Boggs et al., 2009). The ambiguity appears to stem from the types of assays used to assess self-incompatiblity, and how self-incompatibility is defined; from a very narrow temporal window (Nasrallah et al., 2002) or flower age (Tsuchimatsu et al., 2010) to perhaps a fully self-incompatible flower. The defining purpose of self-incompatibililty is to prevent inbreeding; thus, it would have been clearer if seed set was measured consistently in these studies. Nevertheless, these data suggest that there are some inherent differences between some of the A. thaliana ecotypes with regards to the strength of the reconstituted self-incompatibility.

Arabidopsis species diverged 20-40 Mya from Brassica species (Franzke et al., 2011), and it has been proposed that $A$. thaliana uses a different signaling pathway downstream of SRK to Brassica species (Rea et al., 2010; Kitashiba et al., 2011). However, to date, no candidates have been identified for this proposed pathway. Thus, we were interested in assessing the contributions of ARC1 to self-incompatibility in Arabidopsis species. $A R C 1$ was identified in the A. lyrata genome sequence and determined to be deleted in the A. thaliana Col-0 and C24 ecotypes (Kitashiba et al., 2011; Indriolo et al., 2012). ARC1 is expressed in A. lyrata stigmas, but also expressed at lower levels in other tissues (Indriolo et al., 2012). Given that some ecotypes such as Wei-1 and Old-1 contained a functional SRK gene (Tsuchimatsu etal., 2010), we investigated whether an intact $A R C 1$ gene was present in any $A$. thaliana ecotypes. Despite screening 357 different A. thaliana ecotypes, all ecotypes carried the same deletion resulting in a non-functional $A R C 1$ gene. Thus, the ARC1 deletion likely occurred before the different $S R K$ and SCR/SP11 inactivating mutations (since some ecotypes still carried functional SCR or SRK genes; Indriolo et al., 2012). Further analyses on sequenced genomes from other self-compatible Brassicaceae species revealed that in several self-compatible species, $A R C 1$ was non-functional due to large deletions or smaller mutations (Indriolo et al., 2012). As well, self-incompatible species carried intact copies of the ARC1 gene. ARC1's function was then specifically examined in selfincompatible A. lyrata to determine if it was necessary for the rejection of self-pollen. $A R C 1$ expression was knocked down by transforming an ARC1 RNAi construct into A. lyrata resulting in transgenic plants that were no longer able to fully reject selfpollen when self-pollinated. In addition to the observed pollen grain adhesion and pollen tube growth, self-pollinations for transgenic ARC1 RNAi A. lyrata resulted in seed set. In contrast, wild-type self-incompatible $A$. lyrata pollinations resulted in a rejection of all self-pollen and a complete absence of seeds. These results conclusively showed that $A R C 1$ is required for the complete rejection of self-incompatible pollen in A. lyrata, and supported a conserved role for ARC1 downstream of the SRK in both Brassica and Arabidopsis species (Indriolo et al., 2012). It is important to note though that these results do not preclude the presence of other downstream signaling proteins as only a partial breakdown of self-incompatibility was achieved in both studies (Stone etal., 1999; Indriolo et al., 2012).

Most recently, we have completed the reciprocal part of this research and tested ARC1's role in A. thaliana by transforming A. lyrata $A R C 1$ and $B$. napus $A R C 1$ along with the A. lyrata SCRb and $S R K b$ transgenes (Indriolo et al., 2014). Two ecotypes reported to have differing results when transformed with A. lyrata SCRb and 
$S R K b$ were selected: Col-0, which remained self-compatible, and Sha, which became self-incompatible (Nasrallah et al., 2004; Boggs et al., 2009). The main question examined was whether transforming $A R C 1$, along with $S C R b-S R K b$, into these two ecotypes resulted in a stronger self-incompatibility trait. We observed that transgenic $S C R b-S R K b$ Col-0 remained self-compatible as previously reported, but the addition of $A R C 1$ with $S C R b-S R K b$ in Col-0 resulted in clear self-incompatibility. The Sha ecotype was previously reported to display a self-incompatible phenotype with the expression of $S C R b-S R K b$ alone, and we did identify some lines that displayed a moderate self-incompatibility trait. However, we found that the addition of $A R C 1$ resulted in a more robust and stable self-incompatible phenotype with the near complete rejection of self-pollen and little or no seed set (Indriolo et al., 2014). The expression of $A R C 1$ did not affect the levels of SCRb and $S R K b$ transcripts, demonstrating that ARC1 functioned at the protein level as a direct component of the self-incompatibility signaling pathway (Indriolo et al., 2014). Interestingly, we observed an approach herkogamy trait in the transgenic A. thaliana SCRb$S R K b-A R C 1$ plants, where the stigma was positioned above the anthers to avoid self-pollination. Because of this, manual pollinations were conducted for all the analyses to bypass this additional pollen avoidance trait. Overall, the results of these transgenic experiments clearly showed that the addition of either A. lyrata $A R C 1$ or $B$. napus $A R C 1$ with $S C R b-S R K b$ led to stronger selfincompatibility phenotype in both the Col-0 and Sha ecotypes (Indriolo etal., 2014). Furthermore, both A. lyrata ARC1 and B. napus ARC1 exhibited a matching phenotype; thus despite the 20-40 Mya of divergence between A. lyrata and B. napus, the ARC1 protein retained a conserved function in self-incompatibility.

\section{EX070A1, THE TARGET OF ARC1 IN THE SELF-INCOMPATIBILITY PATHWAY}

To determine how ARC1 may be driving the rejection of selfpollen, a yeast two-hybrid screen was performed to search for potential targets of ubiquitination by ARC1, and Exo70A1 was identified (Samuel et al., 2009). Further experiments showed that Exo70A1, not only interacted with ARC1, but that it could be ubiquitinated by ARC1 in an in vitro assay (Samuel et al., 2009). So how does the ubiquitination of Exo70A1 result in the rejection of self-pollen? Before answering this question, Exo70Al's function in the stigma for compatible pollen responses requires explanation. Exo70A1 has previously been shown to act as a marker for secretory vesicles that are delivered to a target membrane via the exocyst complex (reviewed in He and Guo, 2009; Heider and Munson, 2012; Zárský et al., 2013). In response to compatible pollen, the exocyst complex is proposed to mediate polarized exocytosis by docking secretory vesicles at the stigmatic papillar plasma membrane under the pollen contact point. Observations of RFP:Exo70A1 localized to the plasma membrane in stigmatic papillae of mature flowers supported this hypothesis; that Exo70A1 was localized where it was needed to direct exocyst assembly and delivery of secretory vesicles to facilitate hydration of the compatible pollen grain (Samuel et al., 2009). Both A. thaliana exo70a1-1 mutant stigmas and transgenic $B$. napus plants with a stigma-expressed Exo70A1 RNAi knockdown construct were impaired in accepting wild-type compatible pollen validating this prediction. As well, the stigma-specific expression of RFP:Exo70A1 rescued the stigmatic defect in the exo70a1-1 mutant (Samuel et al., 2009). Recently, Li et al. (2013) proposed that Exo70A1 has a tissue-specific expression pattern and functions in developing tracheary elements; however, these observations are not consistent with other research published on A. thaliana Exo70A1 (reviewed in Zárský et al., 2013). Li et al. (2013) also published that $A$. thaliana exo70a1-1 mutant stigmas did not have a defect in accepting compatible pollen. With $A$. thaliana having dry stigmas and previous studies showing that pollen hydration defects can be rescued by high humidity (Preuss et al., 1993; Hülskamp et al., 1995), we tested pollinations under low and high relative humidity and found that this factor may help to explain the discrepancies between these two studies (Safavian et al., Submitted). At low relative humidity, no pollen grain acceptance or seed set was observed on the exo70a1-1 mutant stigma as we had previously published, but high relative humidity conditions did partially rescue the exo70a1-1 mutant stigma resulting in some compatible pollen acceptance and seed set (Safavian et al., Submitted). Exo70A1's expression in the stigma was also verified by RT-PCR (Safavian et al., Submitted). The requirement of Exo70A1 in the stigmatic papillae for compatible pollen acceptance makes it an excellent target for ARC1 in the self-incompatibility pathway. If Exo70Al's activity is inhibited through ubiquitination by ARC1, then the required secretory activity would be blocked in the stigmatic papilla at the pollen contact site causing pollen grain rejection (Figure 1).

To delve into the idea of secretion being required for compatible pollen acceptance and inhibited for self-incompatible pollen rejection, detailed ultrastructural TEM studies were conducted at different time points following pollinations (Safavian and Goring, 2013; Indriolo et al., 2014). In wild-type compatible pollinations at 10 minutes post-pollination, what appeared to be secretory vesicles were visible at the stigmatic papillar plasma membrane at the point of pollen contact in A. thaliana and A. lyrata (Safavian and Goring, 2013). Intriguingly, $B$. napus stigmatic papillae appeared to use multivesicular bodies (MVBs) instead for secretion at the point of pollen contact. This switch to MVBs may be connected to the presence of a thicker cell wall and perhaps a need for increased secretion (Safavian and Goring, 2013). This observed secretory activity was disrupted in the A. thaliana exo70a1-1 mutant stigmas and the transgenic B. napus Exo70A1 RNAi knockdown stigmas following the application of wild-type compatible pollen (Safavian and Goring, 2013). Thus, these studies indicated that Exo70A1 is necessary for exocytosis in the stigmatic papillae following compatible pollinations for promoting pollen hydration and germination.

Self-incompatible pollinations in B. napus, $A$. lyrata, and transgenic $S C R b-S R K b-A R C 1$ A. thaliana all displayed signs of disrupted secretory activity (Safavian and Goring, 2013; Indriolo et al., 2014). Originally, we had thought that the outcome of disrupted secretory activity would be an accumulation of secretory vesicles/MVBs in the cytoplasm, and some of this was seen in the transgenic $S C R b-S R K b-A R C 1$ A. thaliana. However, in $A$. thaliana and $A$. lyrata stigmatic papillae, autophagy appeared to be induced at $10 \mathrm{~min}$ post-self-incompatible pollination. That is, secretory vesicles (and cytoplasm) were being engulfed by autophagosomes and sent to the vacuole for degradation. This 
resulted in the presence of autophagic vacuoles and autophagic bodies in the stigmatic papillar vacuoles (Safavian and Goring, 2013; Indriolo et al., 2014). Again, B. napus stigmatic papillae showed a different degradation response with MVBs re-directed to the vacuole (Safavian and Goring, 2013). These observations suggest that ARC1 may promote more than just the disruption of Exo70A1-guided secretory activity in self-incompatible pollinations (Figure 1). Interestingly in A. thaliana, an ecotype specific difference was observed with the addition of $A R C 1$ with the $S C R b$ $S R K b$ transgenes. The autophagic response was strongest in the transgenic $S C R b-S R K b-A R C 1$ A. thaliana Sha ecotype and not as clear in the transgenic $S C R b-S R K b-A R C 1$ A. thaliana Col-0 ecotype or the transgenic $S C R b-S R K b$ A. thaliana Sha plants lacking $A R C 1$ (Indriolo et al., 2014). This autophagic response in the transgenic SCRb-SRKb-ARC1 A. thaliana Sha ecotype was more similar to that observed in A. lyrata self-incompatible pollinations, and was correlated with the strong self-incompatibility response that we observed in A. thaliana Sha. Finally, the disruption of vesicle secretion was also observed in the transgenic $S C R b-S R K b A$. thaliana Sha plants lacking $A R C 1$, supporting the previous models that there is an additional (unknown) signaling factor(s) functioning downstream of SRK in the cellular response for self-pollen rejection (Rea et al., 2010; Indriolo et al., 2014).

\section{FUTURE RESEARCH DIRECTIONS}

The phenotypes arising from the knockdown of $A R C 1$ in transgenic ARC1 RNAi A. lyrata (Indriolo et al., 2012) and the addition of $A R C 1$ with $S C R b$-SRKb in transgenic A. thaliana (Indriolo et al., 2014) give strong evidence that the $S R K$ - $A R C 1$ self-incompatibility signaling pathway is conserved between Arabidopsis and Brassica species. Despite the progress made into elucidating the role of ARC1 in the self-incompatibility pathway, more questions remain on how it functions as an E3 ubiquitin ligase to regulate downstream targets. Future research directions should focus on identifying the in vivo protein modifications of ARC1 with Exo70A1 during the self-incompatibility response. If ARC1 ubiquitinates Exo70A1, what is the outcome, redirection of Exo70A1's localization or degradation by the $26 \mathrm{~S}$ proteasome? Secondly, how is autophagy in Arabidopsis or vacuolar targeting of MVBs in $B$. napus induced as part the self-incompatibility response, and how is ARC1 and/or other factors involved in this? Finally, the other unknown signaling factor(s) functioning downstream of SRK needs to be identified and how they function in the cellular response for self-pollen rejection needs to be determined.

\section{AUTHOR CONTRIBUTIONS}

Both Emily Indriolo and Daphne R. Goring conceived, designed and wrote this review.

\section{ACKNOWLEDGMENTS}

Research in Daphne R. Goring's laboratory has been supported by a Discovery Grant from the Natural Sciences and Engineering Research Council of Canada and a Canada Research Chair.

\section{REFERENCES}

Andersen, P., Kragelund, B. B., Olsen, A. N., Larsen, F. H., Chua, N. H., Poulsen, F. M., et al. (2004). Structure and biochemical function of a prototypical Arabidopsis U-box domain. J. Biol. Chem. 279, 40053-40061. doi: 10.1074/jbc.M405057200
Boggs, N. A., Dwyer, K. G., Shah, P., McCulloch, A. A., Bechsgaard, J., Schierup, M. H., et al. (2009). Expression of distinct self-incompatibility specificities in Arabidopsis thaliana. Genetics 182, 1313-1321. doi: 10.1534/genetics.109.102442

Bower, M. S., Matias, D. D., Fernandes-Carvalho, E., Mazzurco, M., Gu, T., Rothstein, S. J., et al. (1996). Two members of the thioredoxin-h family interact with the kinase domain of a Brassica S locus receptor kinase. Plant Cell 8, 1641-1650. doi: 10.1105/tpc.8.9.1641

Cabrillac, D., Cock, J. M., Dumas, C., and Gaude, T. (2001). The S-locus receptor kinase is inhibited by thioredoxins and activated by pollen coat proteins. Nature 410, 220-223. doi: 10.1038/35065626

Castric, V., Bechsgaard, J., Schierup, M. H., and Vekemans, X. (2008). Repeated adaptive introgression at a gene under multiallelic balancing selection. PLoS Genet. 4:e1000168. doi: 10.1371/journal.pgen.1000168

Chantha, S. C., Herman, A. C., Platts, A. E., Vekemans, X., and Schoen, D. J. (2013). Secondary evolution of a self-incompatibility locus in the Brassicaceae genus Leavenworthia. PLoS Biol. 11:e1001560. doi: 10.1371/journal.pbio.1001560 PBIOLOGY-D-12-03507

Chapman, L. A., and Goring, D. R. (2010). Pollen-pistil interactions regulating successful fertilization in the Brassicaceae. J. Exp. Bot. 61, 1987-1999. doi: 10.1093/jxb/erq021

Cui, Y., Bi, Y. M., Brugiere, N., Arnoldo, M., and Rothstein, S. J. (2000). The S locus glycoprotein and the $\mathrm{S}$ receptor kinase are sufficient for self-pollen rejection in Brassica. Proc. Natl. Acad. Sci. U.S.A. 97, 3713-3717. doi: 10.1073/pnas.050480297

Dickinson, H. G. (1995). Dry stigmas, water and self-incompatibility in Brassica. Sex. Plant Reprod. 8, 1-10. doi: 10.1007/BF00228756

Foxe, J. P., Slotte, T., Stahl, E. A., Neuffer, B., Hurka, H., and Wright, S. I. (2009). Recent speciation associated with the evolution of selfing in Capsella. Proc. Natl. Acad. Sci. U.S.A. 106, 5241-5245. doi: 10.1073/pnas.0807679106

Franzke, A., Lysak, M. A., Al-Shehbaz, I. A., Koch, M. A., and Mummenhoff, K. (2011). Cabbage family affairs: the evolutionary history of Brassicaceae. Trends Plant Sci. 16, 108-116. doi: 10.1016/j.tplants.2010.11.005

Giranton, J. L., Dumas, C., Cock, J. M., and Gaude, T. (2000). The integral membrane S-locus receptor kinase of Brassica has serine/threonine kinase activity in a membranous environment and spontaneously forms oligomers in planta. Proc. Natl. Acad. Sci. U.S.A. 97, 3759-3764. doi: 10.1073/pnas.97.7. 3759

Gu, T., Mazzurco, M., Sulaman, W., Matias, D. D., and Goring, D. R. (1998). Binding of an arm repeat protein to the kinase domain of the S-locus receptor kinase. Proc. Natl. Acad. Sci. U.S.A. 95, 382-387. doi: 10.1073/pnas.95.1.382

Guo, Y. L., Bechsgaard, J. S., Slotte, T., Neuffer, B., Lascoux, M., Weigel, D., et al. (2009). Recent speciation of Capsella rubella from Capsella grandiflora, associated with loss of self-incompatibility and an extreme bottleneck. Proc. Natl. Acad. Sci. U.S.A. 106, 5246-5251. doi: 10.1073/pnas.0808012106

Haffani, Y., Gaude, T., Cock, J., and Goring, D. (2004). Antisense suppression of thioredoxin h mRNA in Brassica napus cv. Westar pistils causes a low level constitutive pollen rejection response. Plant Mol. Biol. 55, 619-630. doi: 10.1007/s11103-004-1126-x

Hatakeyama, S., and Nakayama, K. (2003). U-box proteins as a new family of ubiquitin ligases. Biochem. Biophys. Res. Commun. 302, 635-645. doi: 10.1016/S0006-291X(03)00245-6

He, B., and Guo, W. (2009). The exocyst complex in polarized exocytosis. Curr. Opin. Cell Biol 21, 537-542. doi: 10.1016/j.ceb.2009. 04.007

Heider, M. R., and Munson, M. (2012). Exorcising the exocyst complex. Traffic 13, 898-907. doi: 10.1111/j.1600-0854.2012.01353.x

Heslop-Harrison, Y., and Shivanna, K. R. (1977). The receptive surface of the angiosperm stigma. Ann. Bot. 41, 1233-1258.

Hiscock, S., and Allen, A. (2008). Diverse cell signalling pathways regulate pollenstigma interactions: the search for consensus. New Phytol. 179, 286-317. doi: 10.1111/j.1469-8137.2008.02457.x

Hülskamp, M., Kopczak, S. D., Horejsi, T. F., Kihlm, B. K., and Pruitt, R. E. (1995). Identification of genes required for pollen-stigma recognition in Arabidopsis thaliana. Plant J. 8, 703-714. doi: 10.1046/j.1365-313X.1995.08050703.x

Indriolo, E., Safavian, D., and Goring, D. R. (2014). The ARC1 E3 ligase promotes two different self-pollen avoidance traits in Arabidopsis thaliana. Plant Cell doi: 10.1105/tpc.114.122879 [Epub ahead of print].

Indriolo, E., Tharmapalan, P., Wright, S. I., and Goring, D. R. (2012). The ARC1 E3 ligase gene is frequently deleted in self-compatible Brassicaceae species and 
has a conserved role in Arabidopsis lyrata self-pollen rejection. Plant Cell 24, 4607-4620. doi: 10.1105/tpc.112.104943

Ivanov, R., and Gaude, T. (2009). Endocytosis and endosomal regulation of the S-receptor kinase during the self-incompatibility response in Brassica oleracea. Plant Cell 21, 2107-2117. doi: 10.1105/tpc.108.063479

Iwano, M., and Takayama, S. (2012). Self/non-self discrimination in angiosperm self-incompatibility. Curr. Opin. Plant Biol. 15, 78-83. doi: 10.1016/j.pbi.2011.09.003

Kachroo, A., Schopfer, C. R., Nasrallah, M. E., and Nasrallah, J. B. (2001). Allele-specific receptor-ligand interactions in Brassica selfincompatibility. Science 293, 1824-1826. doi: 10.1126/science.1062509 293/ $5536 / 1824$

Kakita, M., Murase, K., Iwano, M., Matsumoto, M., Watanabe, M., Shiba, H., et al. (2007a). Two distinct forms of the M-locus protein kinase localize to the plasma membrane and interact directly with the S-locus receptor kinase to transduce self-incompatibility signaling in Brassica rapa. Plant Cell 19, 3961-3973. doi: 10.1105/tpc.106.049999

Kakita, M., Shimosato, H., Murase, K., Isogai, A., and Takayama, S. (2007b). Direct interaction between the S-locus receptor kinase and M-locus protein kinase involved in Brassica self-incompatibility signaling. Plant Biotechnol. 24, 185-190. doi: 10.5511/plantbiotechnology.24.185

Kemp, B. P., and Doughty, J. (2007). S cysteine-rich (SCR) binding domain analysis of the Brassica self-incompatibility S-locus receptor kinase. New Phytol. 175, 619-629. doi: 10.1111/j.1469-8137.2007.02126.x

Kitashiba, H., Liu, P., Nishio, T., Nasrallah, J. B., and Nasrallah, M. E. (2011) Functional test of Brassica self-incompatibility modifiers in Arabidopsis thaliana. Proc. Natl. Acad. Sci. U.S.A. 108, 18173-18178. doi: 10.1073/pnas.11152 83108

Koegl, M., Hoppe, T., Schlenker, S., Ulrich, H., Mayer, T., and Jentsch, S. (1999). A novel ubiquitination factor, $\mathrm{E} 4$, is involved in multiubiquitin chain assembly. Cell 96, 635-644. doi: 10.1016/S0092-8674(00)80574-7

Kusaba, M., Dwyer, K., Hendershot, J., Vrebalov, J., Nasrallah, J. B., and Nasrallah, M. E. (2001). Self-incompatibility in the genus Arabidopsis: characterization of the $\mathrm{S}$ locus in the outcrossing $A$. lyrata and its autogamous relative A. thaliana. Plant Cell 13, 627-643. doi: 10.1105/tpc.13.3.627

Li, S., Chen, M., Yu, D., Ren, S., Sun, S., Liu, L., et al. (2013). EXO70A1-mediated vesicle trafficking is critical for tracheary element development in Arabidopsis. Plant Cell 25, 1774-1786. doi: 10.1105/tpc.113.112144

Liu, J., Li, W., Ning, Y., Shirsekar, G., Cai, Y., Wang, X., et al. (2012). The UBox E3 ligase SPL11/PUB13 is a convergence point of defense and flowering signaling in plants. Plant Physiol. 160, 28-37. doi: 10.1104/pp.112.199430 pp.112. 199430

Lu, D., Lin, W., Gao, X., Wu, S., Cheng, C., Avila, J., et al. (2011). Direct ubiquitination of pattern recognition receptor FLS2 attenuates plant innate immunity. Science 332, 1439-1442. doi: 10.1126/science.1204903 332/ $6036 / 1439$

Mbengue, M., Camut, S., De Carvalho-Niebel, F., Deslandes, L., Froidure, S., KlausHeisen, D., et al. (2010). The Medicago truncatula E3 ubiquitin ligase PUB1 interacts with the LYK3 symbiotic receptor and negatively regulates infection and nodulation. Plant Cell 22, 3474-3488. doi: 10.1105/tpc.110.075861

Mudgil, Y., Shiu, S. H., Stone, S. L., Salt, J. N., and Goring, D. R. (2004). A large complement of the predicted Arabidopsis ARM repeat proteins are members of the U-box E3 ubiquitin ligase family. Plant Physiol. 134, 59-66. doi: 10.1104/pp.103.029553

Murase, K., Shiba, H., Iwano, M., Che, F. S., Watanabe, M., Isogai, A., etal. (2004). A membrane-anchored protein kinase involved in Brassica self-incompatibility signaling. Science 303, 1516-1519. doi: 10.1126/science. 1093586

Naithani, S., Chookajorn, T., Ripoll, D. R., and Nasrallah, J. B. (2007). Structural modules for receptor dimerization in the S-locus receptor kinase extracellular domain. Proc. Natl. Acad. Sci. U.S.A. 104, 12211-12216. doi: 10.1073/pnas.0705186104

Nasrallah, M. E., Liu, P., and Nasrallah, J. B. (2002). Generation of self-incompatible Arabidopsis thaliana by transfer of two S locus genes from A. lyrata. Science 297, 247-249. doi: 10.1126/science.1072205

Nasrallah, M. E., Liu, P., Sherman-Broyles, S., Boggs, N. A., and Nasrallah, J. B. (2004). Natural variation in expression of self-incompatibility in Arabidopsis thaliana: implications for the evolution of selfing. Proc. Natl. Acad. Sci. U.S.A. 101, 16070-16074. doi: 10.1073/pnas.0406970101

Paetsch, M., Mayland-Quellhorst, S., and Neuffer, B. (2006). Evolution of the self-incompatibility system in the Brassicaceae: identification of S-locus receptor kinase (SRK) in self-incompatible Capsella grandiflora. Heredity 97, 283-290. doi: 10.1038/sj.hdy.6800854

Preuss, D., Lemieux, B., Yen, G., and Davis, R. W. (1993). A conditional sterile mutation eliminates surface components from Arabidopsis pollen and disrupts cell signaling during fertilization. Genes Dev. 7, 974-985. doi: 10.1101/gad.7.6.974

Rea, A. C., Liu, P., and Nasrallah, J. B. (2010). A transgenic self-incompatible Arabidopsis thaliana model for evolutionary and mechanistic studies of crucifer self-incompatibility. J. Exp. Bot. 61, 1897-1906. doi: 10.1093/jxb/ erp393

Safavian, D., and Goring, D. R. (2013). Secretory activity is rapidly induced in stigmatic papillae by compatible pollen, but inhibited for self-incompatible pollen in the Brassicaceae. PLOS ONE 8:e84286. doi: 10.1371/journal.pone. 0084286

Salt, J. N., Yoshioka, K., Moeder, W., and Goring, D. R. (2011). Altered germination and subcellular localization patterns for PUB44/SAUL1 in response to stress and phytohormone treatments. PLOS ONE 6:e21321. doi: 10.1371/journal.pone.0021321 PONE-D-11-06319

Samuel, M. A., Chong, Y. T., Haasen, K. E., Aldea-Brydges, M. G., Stone, S. L., and Goring, D. R. (2009). Cellular pathways regulating responses to compatible and self-incompatible pollen in Brassica and Arabidopsis stigmas intersect at Exo70A1, a putative component of the exocyst complex. Plant Cell 21, 2655-2671. doi: 10.1105/tpc.109.069740

Samuel, M. A., Mudgil, Y., Salt, J. N., Delmas, F., Ramachandran, S., Chilelli, A., et al. (2008). Interactions between the S-domain receptor kinases and AtPUB-ARM E3 ubiquitin ligases suggest a conserved signaling pathway in Arabidopsis. Plant Physiol. 147, 2084-2095. doi: 10.1104/pp.108.123380

Samuel, M. A., Salt, J. N., Shiu, S. H., and Goring, D. R. (2006). Multifunctional arm repeat domains in plants. Int. Rev. Cytol. 253, 1-26. doi: 10.1016/S00747696(06)53001-3

Samuel, M. A., Tang, W., Jamshed, M., Northey, J., Patel, D., Smith, D., etal. (2011). Proteomic analysis of Brassica stigmatic proteins following the self-incompatibility reaction reveals a role for microtubule dynamics during pollen responses. Mol. Cell. Proteomics 10, M111.011338. doi: 10.1074/mcp.M111.011338

Schierup, M. H., Mable, B. K., Awadalla, P., and Charlesworth, D. (2001). Identification and characterization of a polymorphic receptor kinase gene linked to the self-incompatibility locus of Arabidopsis lyrata. Genetics 158, 387-399.

Schopfer, C. R., Nasrallah, M. E., and Nasrallah, J. B. (1999). The male determinant of self-incompatibility in Brassica. Science 286, 1697-1700. doi: 10.1126/science.286.5445.1697

Schulman, B. A., and Chen, Z. J. (2005). Protein ubiquitination: CHIPping away the symmetry. Mol. Cell. 20, 653-655. doi: 10.1016/j.molcel.2005. 11.019

Seo, D. H., Ryu, M. Y., Jammes, F., Hwang, J. H., Turek, M., Kang, B. G., et al. (2012). Roles of four Arabidopsis U-box E3 ubiquitin ligases in negative regulation of abscisic acid-mediated drought stress responses. Plant Physiol. 160, 556-568. doi: 10.1104/pp.112.202143

Shimosato, H., Yokota, N., Shiba, H., Iwano, M., Entani, T., Che, F., et al. (2007). Characterization of the SP11/SCR high-affinity binding site involved in self/nonself recognition in Brassica self-incompatibility. Plant Cell 19, 107-117. doi: 10.1105/tpc.105.038869

Silva, N. F., Stone, S. L., Christie, L. N., Sulaman, W., Nazarian, K. A. P., Burnett, L. A., et al. (2001). Expression of the $\mathrm{S}$ receptor kinase in self-compatible Brassica napus cv. Westar leads to the allele-specific rejection of self-incompatible Brassica napus pollen. Mol. Genet. Genomics 265, 552-559. doi: 10.1007/s004380 100446

Stegmann, M., Anderson, R. G., Ichimura, K., Pecenkova, T., Reuter, P., Zarsky, V., et al. (2012). The ubiquitin ligase PUB22 targets a subunit of the exocyst complex required for PAMP-triggered responses in Arabidopsis. Plant Cell 24, 4703-4716. doi: 10.1105/tpc.112.104463

Stone, S., Anderson, E., Mullen, R., and Goring, D. (2003). ARC1 is an E3 ubiquitin ligase and promotes the ubiquitination of proteins during the rejection of selfincompatible Brassica pollen. Plant Cell 15, 885-898. doi: 10.1105/tpc.009845 
Stone, S. L., Arnoldo, M., and Goring, D. R. (1999). A breakdown of Brassica selfincompatibility in ARC1 antisense transgenic plants. Science 286, 1729-1731. doi: 10.1126/science.286.5445.1729

Takasaki, T., Hatakeyama, K., Suzuki, G., Watanabe, M., Isogai, A., and Hinata, K. (2000). The S receptor kinase determines self-incompatibility in Brassica stigma. Nature 403, 913-916. doi: 10.1038/35002628

Takayama, S., Shiba, H., Iwano, M., Asano, K., Hara, M., Che, F. S., et al. (2000) Isolation and characterization of pollen coat proteins of Brassica campestris that interact with $\mathrm{S}$ locus-related glycoprotein 1 involved in pollen-stigma adhesion. Proc. Natl. Acad. Sci. U.S.A. 97, 3765-3770. doi: 10.1073/pnas.97. 7.3765

Takayama, S., Shimosato, H., Shiba, H., Funato, M., Che, F. S., Watanabe, M., et al. (2001). Direct ligand-receptor complex interaction controls Brassica selfincompatibility. Nature 413, 534-538. doi: 10.1038/35097104

Tedder, A., Ansell, S. W., Lao, X., Vogel, J. C., and Mable, B. K. (2011). Sporophytic self-incompatibility genes and mating system variation in Arabis alpina. Ann. Bot 108, 699-713. doi: 10.1093/aob/mcr157

Tsuchimatsu, T., Suwabe, K., Shimizu-Inatsugi, R., Isokawa, S., Pavlidis, P., Stadler, T., et al. (2010). Evolution of self-compatibility in Arabidopsis by a mutation in the male specificity gene. Nature 464, 1342-1346. doi: 10.1038/nature 08927

Vogelmann, K., Drechsel, G., Bergler, J., Subert, C., Philippar, K., Soll, J., et al. (2012). Early senescence and cell death in Arabidopsis saull mutants involves the PAD4-dependent salicylic acid pathway. Plant Physiol. 159, 1477-1487. doi: $10.1104 /$ pp.112.196220

Wang, H., Lu, Y., Jiang, T., Berg, H., Li, C., and Xia, Y. (2013). The Arabidopsis Ubox/ARM repeat E3 ligase AtPUB4 influences growth and degeneration of tapetal cells, and its mutation leads to conditional male sterility. Plant J. 74, 511-523. doi: $10.1111 /$ tpj.12146

Wiborg, J., O'Shea, C., and Skriver, K. (2008). Biochemical function of a typical and variant A. thaliana U-box E3 ubiquitin-protein ligases. Biochem. J. 413, 447-457. doi: 10.1042/BJ20071568

Yee, D., and Goring, D. R. (2009). The diversity of plant U-box E3 ubiquitin ligases: from upstream activators to downstream target substrates. J. Exp. Bot. 60, 11091121. doi: 10.1093/jxb/ern369

Zárský, V., Kulich, I., Fendrych, M., and Peèenková, T. (2013). Exocyst complexes multiple functions in plant cells secretory pathways. Curr. Opin. Plant Biol. 16, 726-733. doi: 10.1016/j.pbi.2013.10.013

Conflict of Interest Statement: Research in DRG's laboratory was conducted in the absence of any commercial or financial relationships that could be construed as a potential conflict of interest.

Received: 31 January 2014; accepted: 16 April 2014; published online: 05 May 2014 Citation: Indriolo E and Goring DR (2014) A conserved role for the ARC1 E3 ligase in Brassicaceae self-incompatibility. Front. Plant Sci. 5:181. doi: 10.3389/fpls.2014.00181 This article was submitted to Plant Genetics and Genomics, a section of the journal Frontiers in Plant Science.

Copyright (c) 2014 Indriolo and Goring. This is an open-access article distributed under the terms of the Creative Commons Attribution License (CC BY). The use, distribution or reproduction in other forums is permitted, provided the original author(s) or licensor are credited and that the original publication in this journal is cited, in accordance with accepted academic practice. No use, distribution or reproduction is permitted which does not comply with these terms. 